\title{
Significance of Forensic Dentistry in Crime Investigations
}

\author{
Kusum Singal \\ Senior Research Fellow (SRF) CDER, AIIMS, India \\ kusumsingal731@gmail.com
}

\begin{abstract}
Forensic dentistry or forensic odontology is the proper handling, examination and evaluation of dental evidence, which will be then presented in the interest of justice. The evidence that may be derived from teeth is the age (in children) and identification of the person to whom the teeth belong. This review article describes significance of forensic dentistry in crime investigation.
\end{abstract}

Keywords: Forensic dentistry, Age estimation, Sex determination, Mass disaster, DNA typing

\section{INTRODUCTION}

The dental enamel is the hardest tissue in the body, and would thus withstand peri- and post-mortem damages, and so would dental materials adjoined to teeth. Being diverse and resistant to environmental challenges, teeth are considered excellent post-mortem material for identification with enough concordant points to make a meaningful comparison ${ }^{1}$." There are three major areas of activity embracing current forensic odontology namely:

1. The examination and evaluation of injuries to teeth, jaws, and oral tissues resulting from various causes

2. The examination of marks with a view to subsequent elimination or possible identification of a suspect as the perpetrator

3. The examination of dental remains (whether fragmentary or complete, and including all types of dental restorations) from unknown persons or bodies with a view to the possible identification of the latter.

The diversity of dental characteristics is wide, making each dentition unique.

\section{THE MAIN ASPECTS OF FORENSIC DENTISTRY INCLUDE ${ }^{2}$}

1. Dental identification-

i) Identification of unknown

ii) Confirmation of identification

2. Age estimation of an individual from teeth

3. Sex determination

4. Role in mass disasters

5. Role in domestic violence, abuse and neglect

6. Identification from bite marks

7. Identification from lip prints

8. Blood group determination

9. Recent advances

i) DNA typing

ii) Computer assisted dental identification

iii) Digital analysis of bite marks

iv) Digital autopsy 


\section{THE ORAL STRUCTURE FINDINGS THAT CONTRIBUTE TO IDENTIFICATION ARE ${ }^{3}$}

1. Age determination (gross, microscopic, radiologic and surface scanning)

2. Sex determination (dental and skeletal comparison)

3. Racial characteristics

4. Blood group determination

5. Other genetic findings

6. Occupational markings

7. Prosthetic markings

8. Individual habits

9. Tooth alignment (occlusion) and abnormalities

10. Jaw deformities (developmental and post-developmental)

11. Dental therapy (fillings, crowns, bridges and dentures)

12. Dental radiology

13. Microscopic examination

14. Holography( where individual tooth prints can be stored in a computer data files, later to be retrieved for identification purposes, as well as for other kinds of data treatment involving oral diagnosis)

\section{DENTAL IDENTIFICATION}

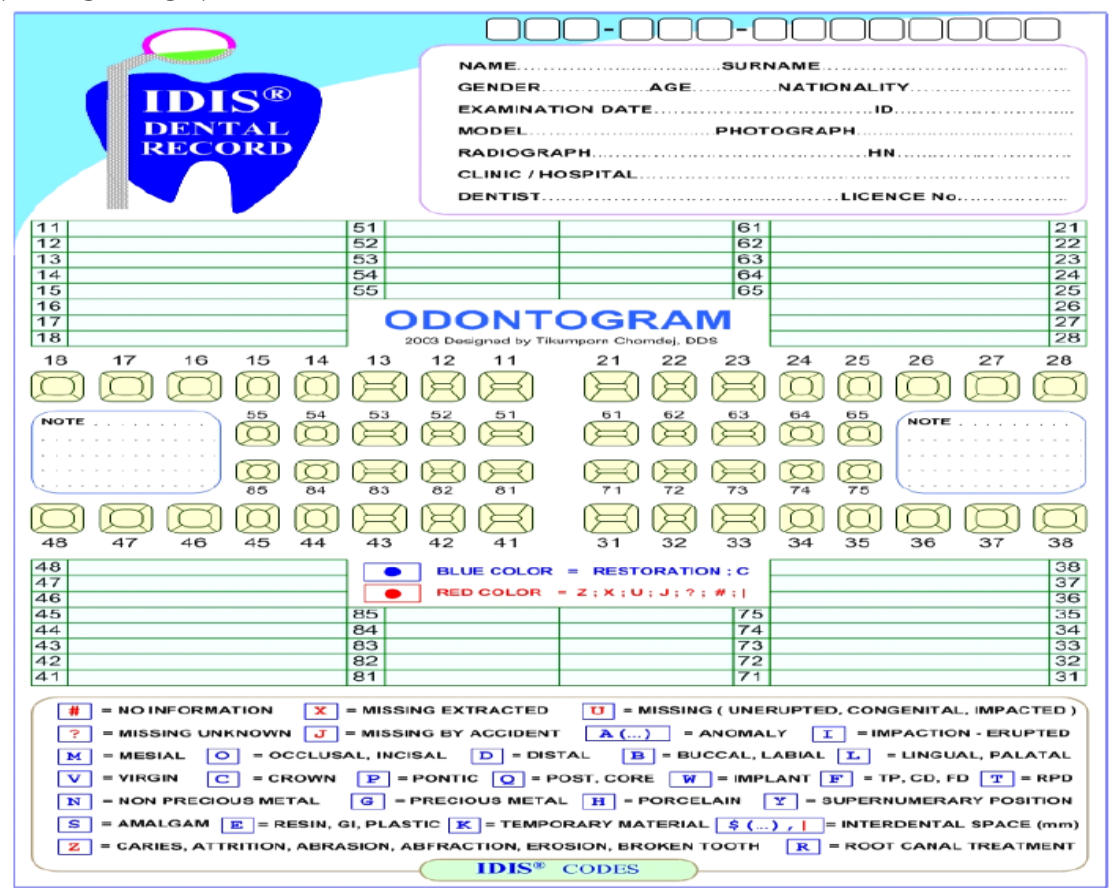

An example of postmortem dental charting ${ }^{6}$

Dental tissue is often preserved indefinitely after death. Complete charting of dentition using FDI (Federation Dentaire International) system or any other nomenclature should be done. Type of dentition (either permanent or deciduous) and surfaces of teeth involved should be evaluated. Bitewing and periapical radiographs are routinely used. Dental impressions like silicones and alginate are used to record bite mark evidence and rugae pattern. Teeth, periodontal tissues and normal anatomical features are assessed in comparative dental identification. Odontograms (symbolic pictorial description of dentition) form a basic outline to compare dental characteristics at the simplest level ${ }^{4}$.The forensic dentist produces the postmortem record by careful charting and written descriptions of the dental structures and radiographs. If the antemortem records are available at this time, postmortem radiographs should be taken to replicate the type and angle of these. Radiographs should be marked with a rubber dam punch to indicate antemortem and postmortem to prevent confusion - one hole for antemortem films and two holes for postmortem films. Once the postmortem record is complete, a comparison between the two records can be carried out. A 
methodical and systematic comparison is required, examining each tooth and surrounding structures in turn. While dental restorations figure significantly in the identification process, many other oral features are assessed, and these play an increasingly important role in those individuals with minimal restorations ${ }^{5}$.

A range of conclusions can be reached when reporting a dental identification. The American Board of

Forensic Odontology recommends that these should be limited to the following four conclusions 5 .

- Positive Identification: The antemortem and postmortem data match in sufficient detail, with no unexplainable discrepancies, to establish that they are from the same individual.

- Possible Identification: The antemortem and postmortem data have consistent features but, because of the quality of either the postmortem remains or the antemortem evidence, it is not possible to establish identity positively.

- Insufficient Evidence: The available information is insufficient to form the basis for a conclusion.

- Exclusion: The antemortem and postmortem data are clearly inconsistent.

When antemortem dental records are unavailable and other methods of identification are not possible, the forensic dentist can assist in limiting the population pool to which the deceased is likely to belong and thus increase the likelihood of locating antemortem dental records. This process is known as postmortem dental profiling. The information from this process will enable a more focused search for antemortem records. A postmortem dental profile will typically provide information on the deceased's age, ancestry background, sex and socio-economic status. In some instances it is possible to provide additional information regarding occupation, dietary habits, habitual behaviours and occasionally on dental or systemic diseases 5 .

\section{Cheiloscopy (EXAMination OF LiP PRINTS)}

The external surface of the lip has numerous elevations and depressions that form a characteristic pattern, referred to as lip prints. Lip prints can be obtained at the crime scene from clothing, cups, glasses, cigarettes, windows, and doors. Using lip prints for personal identification in forensic odontology is an accepted method in the criminal justice system worldwide. Impressions are made from the middle portion of the lower lip, an area always visible in any trace made, and the characteristic patterns are studied. The various patterns identified include vertical, intersected, branched, reticular, and undetermined. The anatomical landmarks of the lip include chelion (the lateral most point in mouth opening), stomion (the contact of upper and lower lips in mid-sagittal plane), and labrale superius and labrale inferius (the highest and lowest points of upper and lower lip margins in the mid-sagittal plane, respectively).Various factors can alter lip print recording. Lip prints have to be obtained within 24 hours of the time of death to prevent erroneous data that would result from postmortem alterations of lip. Lip print pattern depends on whether the mouth is opened or closed. In closed-mouth position lip exhibits well-defined grooves, whereas in open position the grooves are relatively ill defined and difficult to interpret. Any pathology of the lip such as mucocele or any postsurgical alteration of the lip can change the lip print pattern. Also, loss of support due to loss of anterior teeth can cause changes in lip prints. Any debris or fluid on the lip surface, application of a thick layer of lipstick, or over stretching of cellophane tape can alter lip print recording. Although lip prints are unique to an individual, when the lines are not clear, individual identification based on this trace is extremely difficult unless the trace contains more individual characteristics like scars, cleft s, etc ${ }^{7}$.In 1967 Santos 16 was the first person to classify lip grooves He divided them in to four types namely $^{8}$ :

1. Straight line

2. Curved line

3. Angled line

4. Sine-shaped line 


\section{TSUCHIHASHI YT AND SUZUKI T (1968 TO 1971)}

Examined 1364 persons at the department of forensic odontology at Tokyo University and established that the arrangement of lines on the red part of the human lips is individual and unique for each human being'.

Suzuki et al divided them into eight broad categories as:

Type I- a clear cut groove running vertically

Type II- Partial length groove of Type I

Type III- Branched Groove

Type IV- Intersected Groove

Type V- Reticular Pattern

\section{Type VI- Other Pattern}

Sivapathasundharam B, Prakash PA and Sivakumar G (2001) aimed to find out the incidence of the different patterns in 200 individuals. They found that the intersecting pattern is predominately seen $(41.33 \%)$ and least commonly seen pattern is reticular $(10.71 \%)$. Even though these lines and furrows present both in the upper lip and lower lip from one corner of the mouth to the corner, only the middle portion in the lower lip is taken into account, since this portion is always visible in any traces. Because lips are a frequent site of any traumatic injury, due to the inherent bi-maxillary protrusion of teeth in Dravidian, it may lead to scarring and development of mucocele, which may alter the pattern. So a longitudinal study is warranted to note the changes in the lip due to trauma and other pathological lesions like salivary cysts, neoplasms, chelitisglandularis and iatrogenic caused that is plastic surgery of other surgical procedures like lip shaving and lip stripping to treat various developmental lip abnormalities and some mucosal lesions ${ }^{9}$

Rachana V. Prabhu, AjitDinkar and VishnudasPrabhu(2013) defined a standard method or the analysis of lip prints. Lip prints of 100 students were taken using Scotch tape without any distortion. These prints were then scanned for the digital analysis. UsingAdobe Photoshop 7 software an attempt was made to trace each and every line using Suzuki and Tsuchihashi's classification. Weighted value scoring system was used to check for the uniqueness of the lip prints. Results: No two lip prints had exactly matching scores in all four Quadrants. No statistically significant difference was found in the change of pattern in lip print images collected after 12 months from the same individual. With digital analysis, up to 15 lines were traceable in $66 \%, 71 \%, 52 \%$ and $51 \%$ of Quadrant I,II, III and IV respectively. It was concluded that the digital method of analyzing lip print images serves better visualization, ease in identification and recording of the lip print pattern therefore Weighted Value Scoring system can be considered as a standard method for determining the uniqueness of the lip prints $^{10}$.

\section{AmeloglyPhics (Examination OF TOOTh PRints)}

Ameloblasts lay down the enamel rods in an undulating and intertwining path. This is reflected on the outer surface of the enamel as patterns of the ends of a series of adjacent enamel rods. This study of the enamel rod end patterns is termed as ameloglyphics by Manjunath et al.(2014) and could aid as an identification tool in decomposed or burned bodies as enamel can resist decomposition. This study needs antemortem records. Though enamel is the hardest mineralized substance in human body, the enamel surface is usually subjected to micro and macrowearing. Fractured, decayed, attrited, abraded, and eroded teeth cannot be included in this method. Ameloglyphics is still in its infancy and whether the tooth prints are the same at different depths of enamel has to be evaluated with further studies ${ }^{7}$.

\section{RUGOSCOPY}

Rugoscopy is an identification technique based on the study and analysis of the number, shape, length, direction and merging pattern of the palatal ridges or rugae (rugosities). Table 2 shows the rugae classification proposed by Lysell and posteriorly modified by Thomas and Kotze ${ }^{11}$.

The palatal rugae are anatomical ridges, wrinkles or folds located on the anterior portion of the palate, immediately posterior to the upper anterior teeth and the incisive papilla, on either side of the midline. 
The fact that the rugae are present for life, starting from the third month of intrauterine development; are unique to each individual (including twins); and are relatively well protected by the lips, teeth, Bichat's fat pad and the maxillary bones, implies that they are less affected by decomposition and incineration. As a result, the palatal rugae are studied as a method of identification, comparable to the finger prints of the individual ${ }^{11}$.

In a latest study by Rajender G patil (2013), efficiency of computerized software for forensic identification by photographic superimposition of palatal photographs obtained from casts was carried out. In this study two sets of Alginate impressions were made from the upper arches of 100 individuals (50 males and 50 females) with one month interval in between and the casts were poured. All the teeth except the incisors were removed to ensure that only the palate could be used in identification process. In one set of the casts, the palatal rugae were highlighted with a graphite pencil. All the 200 casts were randomly numbered, and then, they were photographed with a 10.1 Mega Pixel Kodak digital camera using standardized method. Using computerized software, the digital photographs of the models without highlighting the palatal rugae were overlapped over the images (transparent) of the palatal rugae with highlighted palatal rugae, in order to identify the pairs by superimposition technique. Incisors were remained and used as landmarks to determine the magnification required to bring the two set of photographs to the same size, in order to make perfect superimposition of images. The result of the overlapping of the digital photographs of highlighted palatal rugae over normal set of models without highlighted palatal rugae resulted in $100 \%$ positive identification. This study showed that utilization of palatal photographs is highly viable. The photographic superimposition technique using Adobe Photoshop 8.0 software (used in this study for comparison of palatal rugae) was proved effective for human identification and can be used when antemortem data regarding palatal rugae is provided. This study also concluded that palatal rugae are highly individualistic and play an important role as a complementary method in personal identification $^{12}$.

\section{REFERENCES}

[1] Al-Amad SH : Forensic Odontology ; Smile Dental Journal Volume 4, Issue 1 - 2009 ; 22-24

[2] Ata-Ali. J, Ata-Ali .F : Forensic dentistry in human identification: A review of the literature ; J Clin Exp Dent. 2014;6(2):e162-7.

[3] Karhunen PJ. Neurosurgical vascular complications associated with aneurysm clips evaluated by post-mortem angiography. Forensic SciInt1991; 51:13-22.

[4] Davson H. The Physiology of the eye. Boston 1963; 2:856-98.

[5] Pretty A and Sweet D: A look at forensic dentistry - Part 1: The role of teeth in the determination of human identity ; British dental journal, volume 190, no. 7, april 142001 : 359366

[6] Chomdel T, Pankaow W, Choychumroon S. Intelligent Dental Identification System (IDIS) dental records :Med J Vol. 49 No. 1 January 2005

[7] Kavitha B. Limitations in forensic odontology : Journal of Forensic Dental Sciences ;JanuaryJune 2009: Vol $1:$ Issue $1: 8-10$

[8] Reddy L: Lip prints: An Overview in Forensic Dentistry : Journal of Advanced Dental Research Vol II : Issue I: January, 2011

[9] Sivapathasundharam B, Prakash PA and Sivakumar G. Lip prints (Cheiloscopy). IJDR 2001 OctDec; 12 (4): 234-237

[10] Rachana V. Prabhu, Dinkar A, Vishnudas P: Digital method for lip print analysis: A New approach. Journal of Forensic Dental Sciences / July-December 2013 / Vol 5 / Issue 2

[11] Schuller A. Das Rontgengram der Stirnhole: EinHiffsmittelfurdieIdentitasbestimmung von Schadeln, Mschr. Ohrenheilk 1921; 55:1617.

[12] Rajendra G. Patil. Rugoscopy: Human identification by computer- assisted photographic superimposition technique. Journal of Forensic Dental Sciences :July-December 2013 :Vol 5 :Issue 2 